\title{
EEG-Robots and Home Automation
}

\author{
Girish Shashikant Patil \\ Ramrao Adik Institute of Technology, Electronics Engineering, First Year, Nerul, Navi Mumbai, India
}

\begin{abstract}
We always try to make computer more and more emphatic to the user.EEG (electroencephalogram) is a way by which we can achieve effective computing. Through this paper we are proposing a technology in which we can see how we can perform a explicit task with the help of EEG waves and brain computer interface. Brain emits five types of waves -beta, gamma, delta, theta, alpha. This waves can be proved very useful if properly executed. Robot-EEG is the best method to make robots do intuitive interaction tasks.
\end{abstract}

Keywords: BCI-brain computer interface, EEG-electroencephalogram, ADHD- atttention deficiet hyperactive disorder. TGAM

\section{Introduction}

EEG is becoming the hot topic in research sciences. EEG is the way by which computer can actually know our emotions and hence can give us a better feedback every time. The deep study of brain waves (gamma waves, beta waves ,alpha waves ,theta waves and delta waves) has concluded some emotions and feelings at particular frequencies of the waves respectively. By the use of these waves computer can actually know our emotion and state of mind at particular sate and hence can perform a particular task we have assigned to the specific range of waves. But our brain actually emits two waves at least every time but its a fact that the dominant frequency is considered at particular sate of mind. Hence such waves which are less emitted at that time are neglected at that particular time by the computer and hence the signals are filtered. The EEG pet system is used to recognise the emotions which gives computer or robots a rough idea of our emotion at the instant.

Hence this emotions can be used to automate the home.

\section{Home Automation Layout}

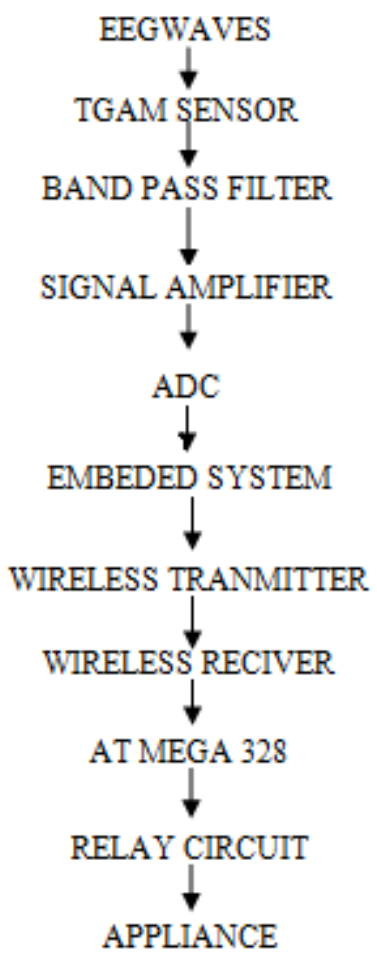

\section{Self Learning Algorithms}

Self learning algorithms are those algorithms which learn by themselves with the use of permutations and combinations. They work like as a sample set in a probability. These algorithms works by deciding the chances at that instant of time. Hence a sample set of actions is needed to be prepared.

By using the above home automation circuit and making some changes in and put self learning algorithm in it. Then the automation could be more effective. the basic idea is to make a robot which can learn and perform actions and tasks done by humans.

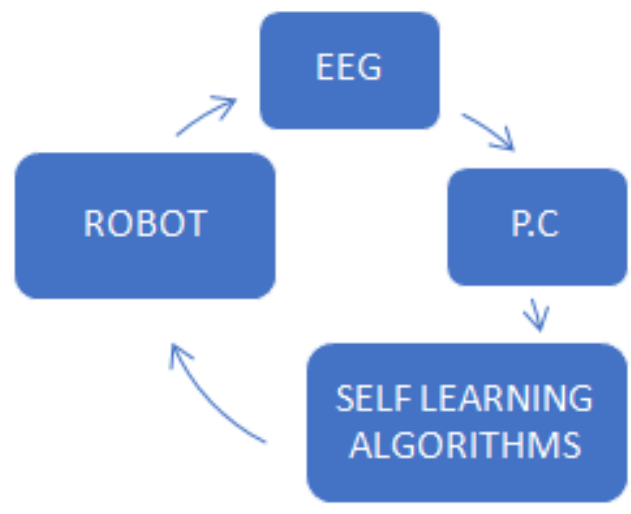

4. Working

TGAM Sensor

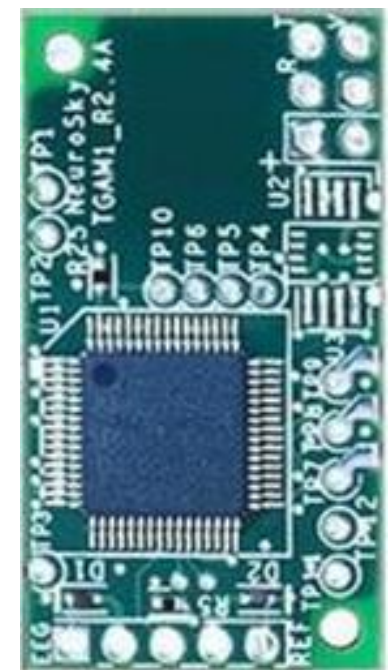

Its is the most important part of EEG. TGAM is the neurosky designed brain wave sensor.

Volume 6 Issue 7, July 2017 www.ijsr.net 


\section{International Journal of Science and Research (IJSR) \\ ISSN (Online): 2319-7064}

Index Copernicus Value (2015): 78.96 | Impact Factor (2015): 6.391

\section{Modulation of Waves}

Waves from the brain are detected using electrodes placed on scalp. There are many ways to place electrodes. The waves detected are then modulated using signal amplifier .

\section{Filter}

Too many waves are emitted at the same time and hence are needed to be filter this happens with the use of filter. For eg:- if gamma waves are emitted dominant then only they are allowed to pass. This is to ensure the explicit tasks at that instant.

\section{Embeded System}

Hence for a specific range of waves some tasks are programmed. This signals are send to wireless transmitter which transmits it. The variety of waves can be used to do the desired task at the particular state of mind.

\section{Examples for Different Waves}

- Gamma waves :- (40- $100 \mathrm{~Hz})$

A PERSON while studying emits gamma waves

Hence if the program is on study mode then robot might be programmed to just give a drink which could helps us in concentration and emit more gamma waves.

\section{- Beta Waves:- (12-40HZ)}

IF a person is emitting beta waves he might be upset or depressed. Hence a hot beverage like tea could be expected from a robots and hence we can accordingly program a robot

\section{- Alpha Waves:-( 8-12 HZ)}

If a person emits more alpha waves he or she might be not able to focous hence we can program a robot which would give a drinks which would help him or her in focusing.

\section{- Theta Waves:- (4-8HZ)}

IF a person is emitting theta waves he is in a deep sleep. Hence AC or fan can be turned on and lights could be turned off.

\section{- Delta Waves (0-4HZ)}

If a man aged above 8 is emitting delta waves he is suffering from ADHD and hence ADHD drugs are expected at this isntant.
Beta ( $\beta$ )
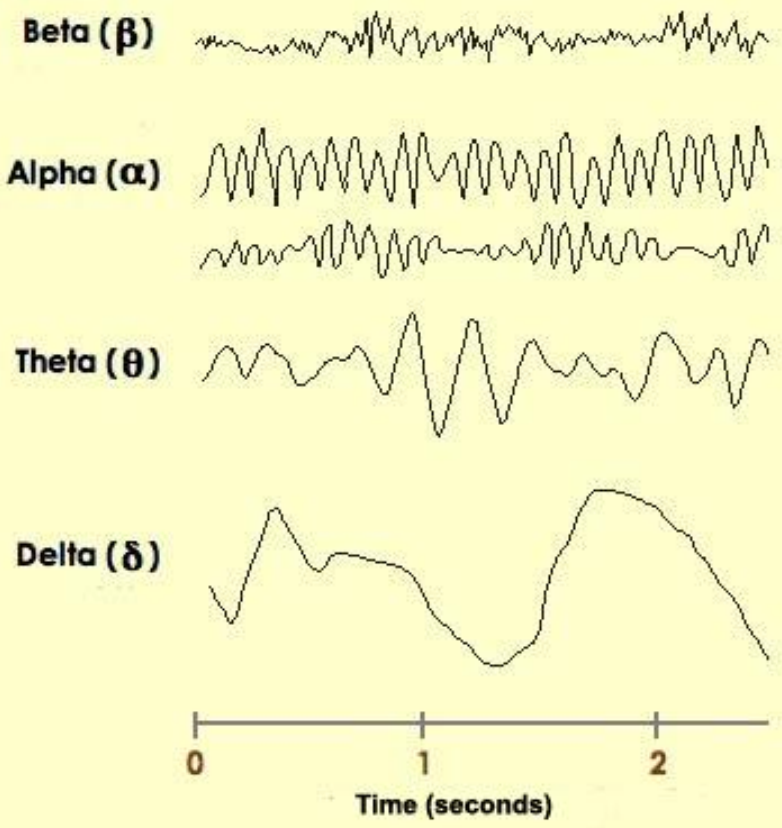

The above can be modified in many various formats and hence desired output can be achieved. We can program the robot for home automation, study mode, paralysed person etc.

\section{Connection Layout for Receiver}

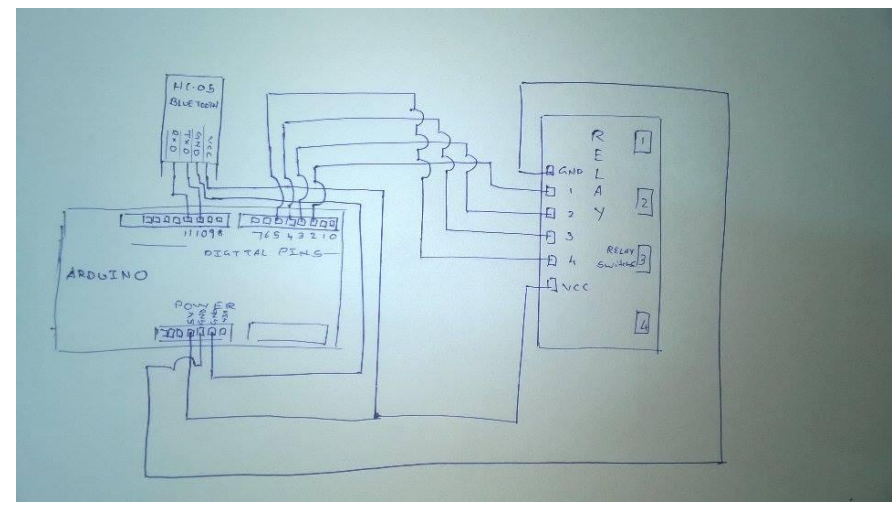

\section{EEG and Robots}

EEG is the way thorough which robots can actually look inside or brain. hence robots can actually understand our emotions and talk to us. This technique would help in human machine interaction. Self learning robots can be made more efficient using the EEG. They could even learn certain actions of human beings by algorithms and hence we can make a quite perfect robot.

By using algorithms a two way talk is achieved . we can just use a pressure and many type of sensor in a robot and hence we can feed and program the robot if robot gets a jerk how is it must react when it must say sorry when can he say it is too hot and as we have our brain waves which it can sense so it can ask us wheater we are upset or depressed or sleepy when he could sense gamma or beta , alpha waves respectively. the above task can be fulfilled if we install temperature Pressure or many other sensor in it. 


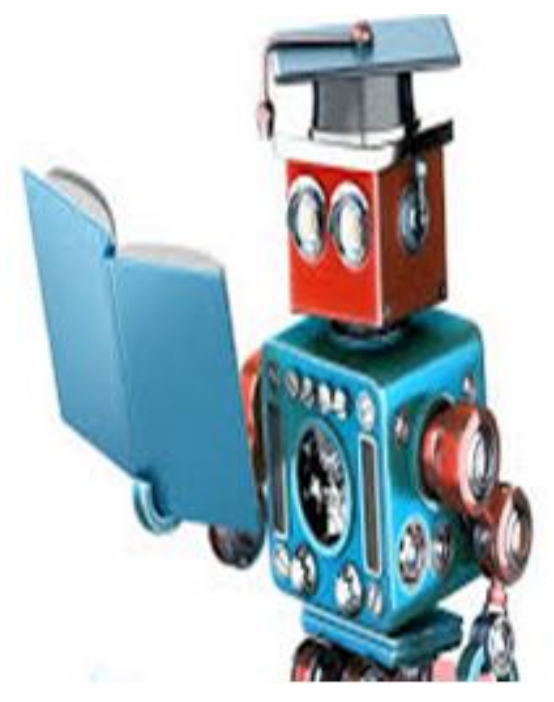

\section{Conclusion}

- Hence EEG waves can be modulated and can be used to communicate with robots.

- And hence we can achieve a two way talk.

- Home automation can be achieved using this proposed system.

- This type of system can be used to help many paralysed patients and ADHD affected persons.

- The self learning robots can be produces with more efficient output

- EEG-home automation-self learning algorithms makes a most efficient robot.

\section{Future Scope}

In future EEG can be used to totally control the robots

\section{Acknowledgement}

I would like to thank DR.S. DHOBLE for his immense support through my project.

\section{References}

[1] S. G. Mason, M. M. Moore Jackson, and G. E. Birch, "A general framework for characterizing studies of brain interface technology," in Annuls of Biomedical Engineering, vol. 33, no II, pp 1653-1670, Nov. 2005.

[2] Vinay Sagar K N, Kusuma $S$ M, "Home Automation Using Internet of Things", International Research Journal of Engineering and Technology (IRJET), Vol. 2, Issue 3, pp: 1965-1970, June 2015.

[3] Apeksha Rani H.M, Prathibha Kiran, "A Novel Method for Analysis of EEG Signals Using Brain Wave Data Analyzer", International Journal of Soft Computing and Engineering (IJSCE), ISSN: 2231-2307, Vol. No. 5, Issue No. 2, pp: 98-100, May 2015.

[4] https://techcrunch.com/2017/03/06/mit-csail-mistakerobot/

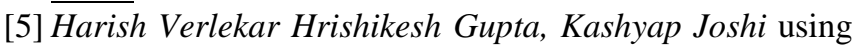
brain computer interface for home automation. International Journal of Engineering Trends and Technology Volume 34 Number 7- April 2016
[6] http://mentalhealthdaily.com/2014/04/15/5-types-ofbrain-waves-frequencies-gamma-beta-alpha-theta-delta/

[7] Andres F. Salazar-Gomez* , Joseph DelPretot , Stephanie Gil广, Frank H. Guenther*, and Daniela Rus $\dagger$ Correcting Robot Mistakes in Real Time Using EEG Signals 